\title{
Time perspective stands out as the time variable that reliably separates anxious and nonanxious individuals
}

\section{Linh-siung Ming*}

Department of Psychology, Jen-Ai Hospital - Taichung, Taiwan.

*Corresponding Author : Linh-siung Ming, Department of Psychology, Jen-Ai Hospital - Taichung, Taiwan.

Received date: June 05,2017;Accepted date : June 15,2017; Published date: June $20,2017$.

Citation for this Article:Linh-siung Ming. Time perspective stands out as the time variable that reliably separates anxious and nonanxious individuals . J Psychology and Mental Health Care . Doi: 10.31579/2637-8892/013

Copyright : (c) 2017 Linh-siung Ming. This is an open-access article distributed under the terms of The Creative Commons Attribution License, which permits unrestricted use, distribution, and reproduction in any medium, provided the original author and source are credited.

\begin{abstract}
Background

It is well-recognized that emotions and emotional disorders may alter the experience of time. Yet relatively little is known about different aspects of psychological time in relation to anxiety. The purpose of the present study was to explore several aspects of temporal processing, including time perspective, prospective and retrospective time estimation, in persons with anxiety symptoms.
\end{abstract}

\section{Methods}

A total of 110 individuals with varying degrees of anxiety participated in two studies. They were assigned to two groups (anxiety-control) based on their scores on anxiety measurements. Participants also completed an inventory of time perspective and several time estimation tasks which were analyzed on a group-level. Depressive symptoms were assessed and used as a covariate in the second study.

\section{Results}

Anxiety was significantly associated with Past Negative and Future Negative time perspectives as measured by the Swedish Zimbardo Time Perspective Inventory (S-ZTPI), even when controlling for the effect of depressive symptoms. No other significant differences were found.

\section{Conclusion}

Exploring time perspective in persons with anxious symptoms may provide important insights into features of anxiety. These findings may offer new ways of conceptualizing anxiety and provide suggestions for treatment strategies.

\section{Keywords}

Anxiety, depression, mental health, temporal processing, time assessment, time estimation, time perspective.

\section{Introduction}

Time is a fundamental part of human existence. We make sense of our daily lives, personal histories and experiences using the temporal frames of past, present and future [1]. Different timing functions also play a vital role in speech, cognition and movement [2].

While objective time progresses linearly in constant units, subjective processing of time may be affected by mental distress or the emotional state of the individual [3-6]. Studying different aspects of temporal processing can thus contribute to a better understanding of the psychological experience of emotions and emotional disorders and underlying mechanisms of temporal processing in normal and clinical populations.

In the present study we aimed to explore different aspects of temporal processing, including time perspective and prospective- and retrospective time estimation in persons with anxiety symptoms. Previous research in the area is rather sparse, and has mainly focused on various time estimation paradigms $[3,7,8]$. Extending this research, our study assessed multiple aspects of psychological time in relation to anxiety; from the basic ability to estimate chronometric time intervals to time perspective, a construct that refers to an individual's habitual approach the past, the present and the future as a personality trait $[1,9]$.

\section{Anxiety and time perspective}

Social psychologist Kurt Lewin [10] regarded time perspective as a dynamic process, where an individual's view of her/his past and anticipation for the future affects current behavior. Zimbardo and Boyd [1] extended on Lewin's view of time perspective and defined it as a cognitive framework that gives meaning and coherence to everyday experiences.
In this context, time perspective is regarded as an important individual difference variable that affects psychological and societal functioning. A central assumption of this definition and operationalization of time perspective is that a subjective well-being reflects a flexible use of the three temporal dimensions (past-present-future) or a balanced time perspective [11]. On the other hand, a biased time perspective, with an overemphasis on one or more of the temporal dimensions (influenced by distress, sickness or trauma) might have direct impact on a person's subjective well-being and social functioning.

To assess time perspective, Zimbardo and Boyd [1] developed the Zimbardo Time Perspective Inventory (ZTPI), a self-report instrument which simultaneously captures an individual's orientations to the past, the present and the future. ZTPI measures time perspective in five different factors (measured through five scales): Past Negative which reflects a negative or aversive view of the past; Past Positive which covers a nostalgic and positive view of one's past; Present Fatalistic which reflects a fatalistic and hopeless view of the present; Present Hedonistic which mirrors an attitude towards the present where pleasure and enjoyment in the now is more important than future outcomes; and finally, the Future factor which reflects a general future orientation, characterized by striving for future goals and rewards.

Anxiety has previously been described as a future oriented emotion [12], involving distress about what may happen [13] and anticipation of future threat or harm [14,15]. Zaleski [16] introduced the concept of Future Anxiety (FA) and regarded it as a personality characteristic where a negative future time perspective precedes the development of anxiety. Taken together, these findings suggest that anxiety is associated with a predominantly future oriented time perspective. 
However, previous research with the ZTPI did not demonstrate a link between the future time perspective and anxiety [1]. In their study, anxiety was instead significantly associated with the Past Negative time perspective and the Present Fatalistic time perspective. We argued that this non-significant relationship was a consequence of the content of the Future scale of the ZTPI being positively biased. The Future scale measures a general goal-oriented and positive outlook on the future, while ignoring negative attitudes and feelings associated with the future, such as fear of the uncertain and worry.

To account for the double emotional valence associated with the future, we utilized the Swedish ZTPI (S-ZTPI) [9] for assessing time perspective. The S-ZTPI extends the original version of the ZTPI by dividing the future into two factors: the Future Positive scale and the Future Negative scale. The Future Positive is largely identical to the Future scale of the ZTPI, while the Future Negative scale is constructed to measure a negative outlook on the future.

\section{Anxiety and time estimation}

Time estimation is involved in everyday human activities, such as estimating whether to brake for a yellow light or not, or estimating the amount of time required to finish a project. Researchers usually distinguish between prospective and retrospective time estimation $[17,18]$. In prospective time estimation, subjects are asked beforehand to estimate time from now into some point in the future. In retrospective time estimation, subjects are asked for unexpected duration estimation after the interval has ended.

Prospective estimates of duration may vary considerably dependent on the cognitive and emotional state of the individual $[3,5]$. For example, in situations such as when we are bored or anticipating an unpleasant event to happen, time subjectively slows down and we tend to overestimate time. On the other hand, when we are engaged in activities that are rewarding or interesting, time appears to pass more quickly and we tend to underestimate time [18].

Cognitive models of prospective time estimation emphasizes the importance of arousal level and attention to time in predicting the direction in prospective time estimation tasks. In these models, a heightened arousal level or increased attention to time may result in longer duration judgments. The relationship between prospective time estimation and anxiety was a fairly popular research topic in the 1960 's and 1970's. These older studies in general found that anxiety was associated with judging time intervals as longer than they chronometrically were, that is, overestimation of time [19-21]. In these studies, overestimation was explained in terms of heightened arousal which increased the pace of a hypothesized internal clock mechanism [22]. In a more recent study, Schweiger and Hiddeman found that in a group of hospitalized cancer patients, high anxiety levels were similarly associated with overestimation of time in a prospective time estimation task [23]. In this study, overestimation was interpreted as resulting from increased attention to time as the psychological distress these patients experienced made it difficult for them to engage in meaningful thoughts and thus, their attention was drawn to time causing longer duration estimates.

In contrast to prospective time estimation, retrospective time estimation relies on incidentally coded temporal information, memory processes and contextual change. To our knowledge, very few studies have investigated retrospective time estimation in relation to anxiety. However, there is some evidence that anxiety is associated with longer retrospective time estimations. For instance, Sarasoon and Stoops [7] found that individuals with high anxiety tended to overestimate time compared to a control group in a situation where they were waiting to perform a test. These findings were explained as resulting from cognitive interference and preoccupation, which made the anxious participants experience that time had passed more slowly.

\section{Purpose of the present study}

In the current study we set out to explore the complex relationship between anxiety and temporal processing, focusing on time perspective, prospective time estimation and retrospective time estimation, in persons with anxiety symptoms.
Consistent with earlier work [20,23] we predicted that persons with anxiety symptoms would show more overe-stimation in prospective time estimation tasks. Similarly, we predicted that anxiety would be associated with overestimation of time in retrospective time estimation [7]. We also expected anxiety to be associated with the Past Negative scale and the Present Fatalistic scale, consistent with previous research [1]. Finally, based on the hypothesis that anxiety is associated with worry and negative anticipation of the future, we hypothesized that anxiety would be associated with higher scores on the Future Negative scale of S-ZTPI.

\section{Methods and results}

\section{Study 1}

\section{Participants}

Fifty-six persons ranging from 19 to 60 years old participated in the study. They were recruited through flyers $(n=32)$ and among clients on a waiting list for psychotherapy at a psychology clinic for trainee therapists in northern Sweden $(n=24)$. Clients at the clinic consist of persons seeking psychotherapy on a voluntary basis, not referred by health care professionals. Potential clients are thoroughly assessed upon admission and persons with more severe psychopathology (such as personality disorders, severe depression or anxiety, eating disorders or schizophrenia) are not admitted to the clinic. The majority of the clients at this clinic have moderate difficulties with anxiety and/or depression.

The participants were divided into two groups; anxiety group-control group based on their scores on the Swedish version of the Symptom Check List 90 (SCL-90) subscales for anxiety disorders (Anxiety, Phobic Anxiety and Obsessive- Compulsive Disorder) [24].

Participants scoring above the threshold for clinical symptoms on any of the anxiety subscales [24] were assigned to the anxiety group $(n=22 ; 17$ females; mean age $=29.7$ years, $S D=11.19$; mean SCL-90 score $=1.45 S D=1.07)$. Fifteen of those were waitlist participants and the remaining seven were participants recruited through flyers. The control group consisted of 34 persons (25 females; mean age $=25.3$ years, $S D=5.31$; mean SCL-90 score $=0.43, S D=0.25$ ). Nine of those were participants from the waiting list while 25 were participants recruited through flyers. The groups differed significantly on their SCL-90 scores, $\mathrm{t}(54)=22.48, p<0.01$, but not on gender distribution $(p=0.64)$ or age $(p=0.17)$. The study was approved by the regional ethics committee at the authors' institution and written informed consent was obtained from all participants.

\section{Procedure and measures}

All participants were tested individually in a quiet room at the psychology clinic and given the same oral instructions. Participants were informed that the aim was to examine individual differences in time perception. Wall clocks were removed and participants were asked to remove wrist watches. The tasks and measurements were presented in the same order as follows.

\section{Time estimation tasks}

Time estimation was measured by a prospective time reproduction task, a prospective time production task and a retrospective time estimation task.

The prospective time reproduction task consisted of computerized task (programmed in e-prime) where a neutral stimulus picture (a "smileyface") appeared on a 15" computer screen at a total of 4 different trials (4, 8,24 and $32 \mathrm{~s}$ ). Each trial was presented twice, in the same random order for each block, except for three practice items $(6,3$ and $9 \mathrm{~s})$ which were presented at the beginning of the session. The participants were asked to reproduce the stimulus by pressing the space bar twice: once to signal the beginning of the reproduction interval and once to signal its end [25].

In the prospective time production task the participants were asked to prospectively judge when two minutes had passed. In order to prevent the participants from sub-vocal counting, they had to perform a distracting task by reading aloud randomly appearing numbers (1-9) from a computer screen.

In the retrospective time estimation task the participants were asked 17 minutes into the experimental session to estimate (in minutes) how much time that had elapsed since the experimental session begun (i.e., "How much time do you think have passed since we first sat down in this room?"). 
The appropriate length of the interval was established after conducting a pilot study and it was chosen for two main reasons. First, the target interval needed to be sufficiently long to produce variation . it needed to extend after the participants had performed the prospective time estimation tasks so that each task would not interfere with the other.

\section{Time perspective self-report}

The Swedish Zimbardo Time Perspective Inventory (S-ZTPI) [9] was used for assessing time perspective. This instrument contains 64 items and participants are asked how characteristic or true the item is for them using a 5-point Likert scale ( $1=v e r y$ uncharacteristic and 5=very characteristic). Internal consistency ranges from 0.84 for the Past Negative scale to 0.70 for the Future Positive scale and test-retest reliability ranges from 0.85 to 0.60 [9].

\section{Data analysis}

Analysis of Variance (ANOVA) was carried out for assessing differences between the groups. The reason for employing a betweengroup design was two-folded. First, many studies in this area (mental distress and time estimation and specifically anxiety and time estimation) have used between-group designs, either based on existing groups or on cut-off scores. A between-group design thus facilitates comparisons with these studies [3,7,21,26-28]. Second, the groups were divided on the basis of clinical levels versus non-clinical levels of anxiety, which further motivates the use of a between-group analysis. Mean ratios were calculated for all the time estimation tasks by dividing the estimated duration in every condition by the actual duration of the same condition. In this procedure, a value $<1$ indicates underestimation and a value $>1$ indicates overestimation of the time interval [28]. There was one missing value in the S-ZTPI data and it was replaced using the multiple imputation function in SPSS.

\section{Results 1}

Table 1 summarizes the main findings of the study. As presented in the table, the between groups analysis (ANOVA) showed that the anxiety group scored significantly higher on the Past Negative scale and the Future Negative scale. There were no significant differences between the groups on any of the duration intervals $(p>0.05)$ in the prospective time reproduction task and both groups tended to underestimate time (mean relative error <1). The same pattern was observed in the prospective time production task, both groups tended to underestimate the time interval. In the retrospective time estimation task, both groups overestimated the time interval slightly. The ANOVA revealed no significant differences between the groups on neither the prospective time production task nor the retrospective time estimation task.

\begin{tabular}{|c|c|c|c|c|c|}
\hline & \multicolumn{2}{|c|}{ Group } & \multirow[b]{3}{*}{$\boldsymbol{F}$} & \multirow[b]{3}{*}{$p$} & \multirow{3}{*}{$\begin{array}{l}\text { Coht } \\
\text { n's d }\end{array}$} \\
\hline & \multirow{2}{*}{\begin{tabular}{|l|} 
Anxiety \\
M (SD) \\
\end{tabular}} & \multirow{2}{*}{\begin{tabular}{|l|} 
Control \\
M (SD) \\
\end{tabular}} & & & \\
\hline & & & & & \\
\hline \multicolumn{6}{|l|}{ S-ZTPI } \\
\hline Past Negative & $2.81(0.69)$ & $2.35(0.53)$ & 8.03 & 0.01 & 0.78 \\
\hline Past Positive & $3.67(0.54)$ & $3.61(0.65)$ & 0.12 & 0.73 & 0.10 \\
\hline Present Fatalistic & $2.60(0.42)$ & $2.36(0.58)$ & 2.59 & 0.11 & 0.44 \\
\hline Present Hedonistic & $3.06(0.54)$ & $3.07(0.53)$ & 0.00 & 0.96 & 0.01 \\
\hline Future Negative & $3.24(0.45)$ & $2.92(0.41)$ & 7.54 & 0.01 & 0.76 \\
\hline Future Positive & $3.51(0.52)$ & $3.61(0.57)$ & 0.41 & 0.52 & 0.18 \\
\hline \multicolumn{6}{|c|}{ Prospective time reproduction } \\
\hline 4 seconds & $0.93(0.17)$ & $0.88(0.16)$ & 1.66 & 0.20 & 0.35 \\
\hline 8 seconds & $0.97(0.11)$ & $0.98(0.11)$ & 0.05 & 0.82 & 0.06 \\
\hline 24 seconds & $0.92(0.14)$ & $0.98(0.13)$ & 2.54 & 0.12 & 0.44 \\
\hline 32 seconds & $0.95(0.08)$ & $0.93(0.11)$ & 0.44 & 0.51 & 0.18 \\
\hline \multicolumn{6}{|c|}{ Prospective time production } \\
\hline 2 minutes & $0.94(0.35)$ & $0.90(0.30)$ & 0.15 & 0.70 & 0.11 \\
\hline \multicolumn{6}{|c|}{ Retrospective time estimation } \\
\hline 17 minutes & $1.03(0.34)$ & $1.02(0.36)$ & 0.00 & 0.98 & 0.01 \\
\hline
\end{tabular}

Table 1 Mean (M), Standard Deviations (SD), F-statistics with $\boldsymbol{p}$-Values and Effect Sizes (Cohen's $\boldsymbol{d}$ ) for Inventories of Time Perspective, Prospective Time Reproduction, Prospective Time Production and Retrospective Time Estimation.

\section{Study 2}

Study 2 was designed to address some of the limitations of the first study. Specifically, we intended to control for possible confounding effects of depression. Anxiety and depression often co-occur and depression is the most commonly comorbid disorder associated with anxiety [29]. Although the relationship between anxiety and depression is well recognized [30], anxiety and depression may nevertheless be associated with different causes and consequences [31]. For instance, they may differ in terms of time perspective. Depressed individuals may be biased towards past orientation, with rumination over past failures and loss, while anxious individuals are more oriented towards the future, with anticipation of future threats and negative outcomes [32].

Our results from Study 1 suggested a link between Future Negative time perspective and anxiety symptoms; however, there was also a relationship between Past Negative time perspective and anxiety symptoms. We argued that depressive symptoms in the participants (specifically in the anxiety group) could partly explain this finding. To further disentangle the relationship between time perspective and anxiety, we assessed depressive symptoms in Study 2. To facilitate differentiation of anxiety symptoms from depression, the measurement of anxiety was changed from SCL-90 to Beck Anxiety Inventory (BAI) [33]. The reason behind this methodological change was that the discriminant validity and sensitivity of the SCL-90 scales may be questionable [24,34]. BAI on the other hand, was specifically developed to provide an anxiety measurement that minimally overlaps with depression [33].

\section{Participants}

Forty-four persons ranging from 18 to 51 years old participated in the study. They were recruited among clients on a waiting list for psychotherapy at a psychology clinic for trainee therapists in northern Sweden $(n=20)$ and through flyers $(n=24)$. The participants were divided into two groups (anxiety group-control group) based on their scores on the Beck Anxiety Inventory (BAI) [33]. Participants scoring above the threshold for mild anxiety $(\mathrm{BAI} \geq 8)$ were assigned to the anxiety group. Participants scoring below this threshold were assigned to the control group. The anxiety group consisted of 19 persons (15 females; mean age $=25.58, S D=5.18$, mean BAI score $=16.11, S D=6.4)$ and the control group consisted of 25 persons (18 females; mean age $=26.36, S D=7.37$, mean BAI score $3.60, S D=2.02$ ). The anxiety group consisted of 11 participants recruited through flyers and 8 waiting list participants. The groups differed significantly on BAI score, $\mathrm{t}(42)=9.2, p<0.01$, but not on gender distribution $(p=0.22)$ or age $(p=0.80)$. Participants were assessed for depressive symptoms using the Beck Depression Inventory-II (BDI) [35]. The study was approved by the regional ethics committee at the authors' institution and written informed consent was obtained from all participants.

\section{Procedure and measures}

All participants were tested individually in a quiet room at the psychology clinic and given the same oral instructions. Participants were informed that the aim was to examine individual differences in time perception. Wall clocks were removed and participants were asked to remove wrist watches. The tasks and measurements were presented in the same order as follows.

\section{Time estimation tasks}

Time estimation was measured using a prospective time production task and a retrospective time estimation task.

The prospective time production task was identical to the task included in Study 1 (see Method 1). In the retrospective time estimation task the subjects were asked 12 minutes into the experimental session to estimate how much time they thought had elapsed since the session begun (i.e., "How much time do you think have passed since we first sat down in this room?"). The rationale behind the target length of the retrospective time interval was the same as in Study 1 (see Method 1).

\section{Time perspective self-report}

Time perspective was assessed with the Swedish Zimbardo Time Perspective Inventory (S-ZTPI; see Method 1). 


\section{Results 2}

As presented in Table 2, the between subjects analysis (ANOVA) showed that the anxiety group differed significantly on the Past Negative and Future Negative scale, with the anxiety group scoring significantly higher on those subscales. As expected, the anxiety group scored significantly higher than the control group on BDI (see Table 2 ). Therefore, in the next step of the analysis, depressive symptoms were included as a covariate. The separate ANCOVA's revealed a remaining effect of anxiety on both the Past Negative, $F$ (1, $42)=6.8, p<0.05$ and the Future Negative scale, $\mathrm{F}(1,42)=5.8, p<0.05$.

\begin{tabular}{|c|c|c|c|c|c|}
\hline & \multicolumn{2}{|c|}{ Group } & \multirow[b]{3}{*}{$\mathbf{F}$} & \multirow[b]{3}{*}{ p } & \multirow{3}{*}{$\begin{array}{l}\text { Cohe } \\
\text { n's d }\end{array}$} \\
\hline & Anxiety & Control & & & \\
\hline & $\mathrm{M}(\mathrm{SD})$ & $\mathrm{M}(\mathrm{SD})$ & & & \\
\hline \multicolumn{6}{|l|}{ S-ZTPI } \\
\hline Past Negative & $3.53(0.71)$ & $2.77(0.55)$ & 15.70 & 0.00 & 1.23 \\
\hline Past Positive & $3.26(0.70)$ & $3.55(0.78)$ & 1.60 & 0.21 & 0.39 \\
\hline Present Fatalistic & $2.67(0.42)$ & $2.52(0.39)$ & 1.64 & 0.21 & 0.40 \\
\hline Present Hedonistic & $3.18(0.54)$ & $3.28(0.58)$ & 0.35 & 0.56 & 0.18 \\
\hline Future Negative & $3.33(0.68)$ & $2.69(0.56)$ & 11.70 & 0.00 & 1.07 \\
\hline Future Positive & $3.44(0.70)$ & $3.36(0.65)$ & 0.17 & 0.68 & 0.13 \\
\hline BDI score & $16.11(6.59)$ & $6.88(5.63)$ & 25.01 & 0.00 & 1.56 \\
\hline \multicolumn{6}{|c|}{ Prospective time production } \\
\hline 2 minutes & $1.02(0.33)$ & $0.91(0.28)$ & 1.35 & 0.25 & 0.36 \\
\hline \multicolumn{6}{|c|}{ Retrospective time estimation } \\
\hline 12 minutes & $1.02(0.27)$ & $0.91(0.29)$ & 1.66 & 0.21 & 0.40 \\
\hline
\end{tabular}

Table 2 : Mean (M), Standard Deviations (SD), F-Statistics with $p$-Values and Effect Sizes (Cohen's $d$ ) for Inventories of Time Perspective, Prospective Time Production, Retrospective Time Estimation and BDI score.

In the prospective time production task, the anxiety group slightly overestimated the time interval, while the control group underestimated the time interval. A similar pattern was observed in the retrospective task, where the anxiety group slightly overestimated the time interval and the control group underestimated the time interval. There were no significant differences in time estimation between the groups on either of the tasks (see Table 2).

\section{Discussion}

The aim of the two studies presented in this article was to investigate several aspects of temporal processing in persons with anxiety symptoms. The results consistently showed that persons with anxiety symptoms are more prone to look at their future with worry and negative anticipation (Future Negative time perspective) and recall their past with regret and aversive feelings (Past Negative time perspective). Although conceptualizations of anxiety focus on the future negative orientation $[12,14]$ our studies show that persons with anxiety are also affected by a Past Negative time perspective. One could argue that these findings only reflect the negative valence of the Future Negative scale and the Past Negative scale. If this was true, we should also have found a significant association with the Present Fatalistic scale since it also has a negative emotional valence.

The past and the future have been linked in previous neuropsychological research. Neuroimaging studies examining the concept of mental time travel [36,37] which refers to the ability to imagine past or future events, suggest that people utilize similar brain areas when thinking about their past and imagining their future. When imagining the future scenarios, people tend to place these scenarios in well-known (past) contexts [37]. This rationale is also supported by studies on patients with lesions or psychopathology associated with episodic memory problems [38]. In these cases, patients exhibit both difficulties in recalling episodic memories, as well as imagining their personal future [38]. For the anxiety group, there are likely negative past experiences in their life histories (for instance a traumatic event responsible for their current anxiety) that influence their future predictions.
It is also however likely that they subjectively evaluate their past negatively (because of current anxiety) which would similarly affect their predictions and views of the future.

We did not find any significant patterns of time estimation deficits related to anxiety in either of our two studies independent of time estimation paradigm (prospective and retrospective) and duration length. These results are though not consistent with past research. [3,7,23]. For prospective time estimation, these findings can be understood in the framework of the attentional gate model (AGM) [17]. The AGM is a widely used cognitive model for explaining prospective time estimation. It poses an internal clock with a pacemaker that emits pulses at a constant rate. When attention is drawn to time, an attentional gate opens, and time units are fed into an accumulator. The number of pulses in the accumulator is then compared with stored representations of time intervals. The association between anxiety and duration overestimation can be explained in two ways according to the AGM [3,23]. Firstly, increased arousal which can be associated with anxiety leads to higher rates of the pacemaker which means a greater accumulation of pulses (or time units) during a given time period [3]. Secondly, anxiety may be associated with less meaningful thoughts and fear of the future, which may lead to an increased attention to time [23] resulting in overestimation of time in prospective time estimation tasks. In the context of AGM, the most probable explanation for our findings is that the samples consisted of persons with overall mild to moderate symptoms of anxiety. That is, the participants were not sufficiently anxious during the experimental session for significantly affecting time estimation.

The lack of significant results in our studies is comparable to the findings by Lennings and Burns [39] concerning prospective time estimation and high self-reported impulsivity in a non-clinical sample of students. Whereas other studies investigating impulsivity in clinical populations have found pronounced overestimations associated with impulsivity $[25,26]$, the former study did not find an association between impulsivity and time estimation. The results of the present studies mirrors this finding as it is not research conducted on severe cases of psychopathology. This means that our results concerning time estimation are probably not generalizable to individuals suffering from severe anxiety.

\section{Conclusions}

Although the present study revealed some interesting findings, it has several limitations. First, the small sample size renders it difficult to detect small group differences, as seen in the time estimation tasks. However, given the small effect sizes for the group differences (see Tables 1 and 2), it is unlikely that we would have found any significant differences even with a larger sample. As discussed in previous sections, these results were more likely due to sample characteristics of the anxiety group. Second, time perspective was self-reported. Future research should include additional measures of time perspective to provide more robust findings, such as the Circles Test [40] or the more recently developed Time relation test [41]. Both of these instruments offer ways to measure aspects of time perspective that differs from self-report questionnaires. In the Circles Test [40], participants are asked to draw three circles illustrating the emphasis they place on each time frame (past, present and future). The Time relation test is developed on the basis of the Circles Test and offers a promising solution to the scoring difficulties associated with the original test [41] In this instrument, participants are offered a set of circles of standardized sizes to choose from. The Time relation test has however so far only been validated in adolescents. Using these types of instruments in combination with the ZTPI may offer more substantial findings and is a fruitful future research area. Finally, the psychometric properties of the ZTPI (and consequently the S-ZTPI) have been criticized [42,43]. Nonetheless, to date, the ZTPI is the preferable option for multidimensional assessment of time perspective in adults and a step forward regarding limitations of previous scales $[1,44]$.

Despite the limitations, our study yielded some interesting findings. First and foremost, time perspective stands out as the time variable that reliably separates anxious and nonanxious individuals. The temporal bias towards negative aspects of the past and the future but not the present reveals interesting features of anxiety and provides some insights into the relationship between time and psychopathology. 
These findings also raise an important question: Does anxiety lead to a biased time perspective or does a time perspective with high degrees of Past Negative and Future Negative lead to increased vulnerability for anxiety? The latter would mean that time perspective is an important variable to consider in preventing mental health problems. This issue, concerning the direction of causality, further highlights the importance of longitudinal studies investigating the relationship between time perspective and symptom level over time. Furthermore, assessing time perspective profiles in persons with anxiety, depression or other conditions of mental distress, may offer a new way of conceptualizing mental distress that could give suggestions for new treatment strategies. Future research should try to further disentangle the links between time perspective-profiles in anxiety, symptom level and possible underlying mechanisms.

\section{References}

1. Zimbardo PG and Boyd JN. Putting time in perspective: A valid, reliable individual differences metric. J Pers Soc Psychol. 1999; 77:1271-1288.

2. Zelaznik HN, Spencer RMC and Ivry RB. Behavioral Analysis of Human Movement Timing. In Grondin S (Ed.), Psychology of Time. Howard House, W L (UK): Emerald 2008; 233-260.

3. Bar-Haim Y, Kerem A, Lamy D and Zakay D. When time slows down: The influence of threat on time perception in anxiety. Cogn Emot. 2010; 24:255-263.

4. Blewett AE. Abnormal subjective time experience in depression. Br J Psychiatry. 1992; 161:195-200.

5. Droit-Volet $S$ and Meck WH. How emotions colour our perception of time. Trends Cogn Sci. 2007; 11:504-13.

6. Van Beek W, Berghuis H, Kerkhof A and Beekman A. Time perspective, personality and psychopathology: Zimbardo's time perspective inventory in psychiatry. Time and Society. 2011; 20:364-374.

7. Sarason IG and Stoops R. Test anxiety and the passage of time. J Consult Clin Psychol. 1978; 46:102-9.| |

8. Tipples J. Negative emotionality influences the effects of emotion on time perception. Emotion. 2008; 8:127-31.

9. Carelli MG, Wiberg B and Wiberg M. Development and construct validation of the Swedish Zimbardo Time Perspective Inventory. Eur J Psychol Assess. 2011; 27:220-227.

10. Lewin K. Field theory in social sciences: Selected theoretical papers. Oxford, England: Harpers. 1951.

11. Wiberg M, Sircova M, Wiberg B and Carelli MG. Operationalizing balanced time perspective in a Swedish sample. International $\mathrm{J}$ Educational and Psychol Assess. 2012; 12:95-107.

12. Barlow DH. Anxiety and its disorders - The nature and treatment of anxiety and panic (2nd ed). New York: The Guilford Press. 2002.

13. Adshead G. The time of our lives: Psychological disorders, time perception and the practice of mindfulness. Eur $\mathrm{J}$ Psychother Counsel. 2013; 15:139-150.

14. Beck AT and Emery G. Anxiety disorders and phobias. New York: Basic Books 2005.

15. MacLeod AK and Byrne A. Anxiety, depression, and the anticipation of future positive and negative experiences. $\mathbf{J}$ Abnorm Psychol. 1996; 105:286-9.

16. Zaleski Z. Future anxiety: Concept, measurement, and preliminary research. Pers Indiv Differ. 1996; 20:165-174.

17. Block RA and Zakay D. Prospective and retrospective duration judgments: A meta-analytic review. Psychon Bull Rev. 1997; 4:184-97.

18. Wittmann M. The inner experience of time. Philos Trans R Soc Lond B Biol Sci. 2009; 364:1955-67.

19. Johnson DT. Trait anxiety, state anxiety, and the estimation of elapsed time. J Consult Clin Psychol. 1968; 32:654-8.

20. Siegman AW. Anxiety, impulse control, intelligence, and the estimation of time. J Clin Psychol. 1962; 18:103-5.
21. Whyman AD and Moos RH. Time perception and anxiety. Percept Mot Skills. 1967; 24:567-70.

22. Gilbert FS, Dillbeck MC, Curran JP and Little LM. The perception of time following stress. Motiv Emotion . 1977; 1:151-163.

23. Wittmann M, Vollmer T, Schweiger $\mathrm{C}$ and Hiddemann W. The relation between the experience of time and psychological distress in patients with hematological malignancies. Palliat Support Care. 2006; 4:357-63.

24. Fridell M, Cesarec Z, Johansson M and Thorsen SM. SCL-90 Swedish validation and standardizatin och the symptom scale [Original title in Swedish: SCL-90 - Svensk normering, standardisering och validering av symtomskalan]. Stockholm: Statens institutions styrelse (SIS) 2002.

25. Carelli MG, Forman H and Mantyla T. Sense of time and executive functioning in children and adults. Child Neuropsychol. 2008; 14:372-86.

26. Berlin HA and Rolls ET. Time perception, impulsivity, emotionality, and personality in self-harming borderline personality disorder patients. J Pers Disord. 2004; 18:358-78.

27. Gil S and Droit-Volet S. Time perception, depression and sadness. Behav Processes. 2009; 80:169-76.

28. Wittmann M, Leland DS, Churan J and Paulus MP. Impaired time perception and motor timing in stimulant-dependent subjects. Drug Alcohol Depend. 2007; 90:183-92.

29. Brown TA, Campbell LA, Lehman CL, Grisham JR and Mancill RB. Current and lifetime comorbidity of the DSM-IV anxiety and mood disorders in a large clinical sample. J Abnorm Psychol. 2001; 110:585-99.

30. Mineka S, Watson D and Clark LA. Comorbidity of anxiety and unipolar mood disorders. Annu Rev Psychol. 1998; 49:377-412.

31. Beuke CJ, Fischer R and McDowall J. Anxiety and depression: why and how to measure their separate effects. Clin Psychol Rev. 2003; 23:831-48.

32. Nolen-Hoeksema S,Wisco BE and Lyubomirsky S. Rethinking Rumination. Perspect Psychol Science. 2008; 3:400-424

33. Beck AT, Epstein N, Brown G and Steer RA. An inventory for measuring clinical anxiety: psychometric properties. J Consult Clin Psychol. 1988; 56:893-7.

34. Holi MM, Sammallahti PR and Aalberg VA. A Finnish validation study of the SCL-90. Acta Psychiatr Scand .1998; 97:42-6.

35. Beck AT and Steer RA. Beck Depression Inventory [Manual, Swedish version]. Fagernes: Psykologiförlaget AB 1996.

36. Addis DR, Wong AT and Schacter DL. Remembering the past and imagining the future: common and distinct neural substrates during event construction and elaboration. Neuropsychologia .2007; 45:1363-77

37. Szpunar KK, Watson JM and McDermott KB. Neural substrates of envisioning the future. Proc Natl Acad Sci U S A. 2007 ; 104:642-7.

38. Schacter DL and Addis DR. The cognitive neuroscience of constructive memory: remembering the past and imagining the future. Philos Trans R Soc Lond B Biol Sci. 2007; 362:773-86. |

39. Lennings $\mathrm{CJ}$ and Burns AM. Time perspective: temporal extension, time estimation, and impulsivity. J Psychol . 1998; 132:367-80.

40. Cottle TJ. The circles test: an investigation of perceptions of temporal relatedness and dominance. J Proj Tech Pers Assess. 1967; 31:58-71.

41. Mello ZR, Finan L and Worrell FC. Introducing an instrument to measure time orientation and time relation in adolescents. $\mathrm{J}$ Adolescence. 2013; 36: 551-563.

42. Andretta JR, Worrell FC Mello ZR, Dixson DD and Baik SH. Demographic group differences in adolescents ' time attitudes. J Adolescence. 2013; 36:289-301

43. Worrell FC and Mello ZR. The reliability and validity of Zimbardo Time Perspective Inventory Scores in academically talented adolescents. Educ Psychol Meas. 2007; 67:487-504.

44. Boniwell I, Osin E, Linley PA and Ivanchenko GV. A question of balance: Time perspective and well-being in British and Russian samples. J Posit Psychol. 2010;5:24-40. 